\title{
DYSCERNE: developing clinical management guidelines for selected dysmorphic syndromes
}

\author{
Pam Griffiths ${ }^{1 *}$, Kate Strong ${ }^{1}$, Sara Gardner ${ }^{1}$, Ruth Day $^{1}$, Caroline Harrison ${ }^{1}$, Kerr Bronwyn ${ }^{1}$, Kay Metcalfe ${ }^{1}$, \\ Han Brunner ${ }^{3}$, Dian Donnai ${ }^{1}$, Bruno Dallapiccola ${ }^{4}$, Koenraad Devriendt ${ }^{2}$, Malgorzata Krajewska-Walasek ${ }^{6}$, \\ Nicole Philip ${ }^{5}$, Jill Clayton-Smith ${ }^{1}$
}

From 5th European Conference on Rare Diseases (ECRD 2010)

Krakow, Poland. 13-15 May 2010

The DYSCERNE Network of Centres of Expertise for dysmorphology (http://www.dyscerne.org), is developing clinical management guidelines for Williams (WS), Angelman (AS), Noonan (NS) and Kabuki (KS) syndromes. An initial scoping exercise identified these conditions as rare, complex, multi-system disorders, for which it was felt that affected patients, families and health/social care professionals would benefit from access to up-to-date evidence based management guidelines.

Published evidence from which to develop management recommendations for these conditions is very limited, and devising a systematic and robust methodology has been challenging. Our approach is based on the Scottish Intercollegiate Guidelines Network (SIGN) method, which we modified placing more emphasis on expert opinion and consensus, whilst maintaining systematic rigour and transparency of processes.

The development process includes:

- Identification of key management issues by guideline group leaders.

- Targeted, systematic literature searches using PubMed.

- Review, identification and grading of results by panel of invited experts.

- Consensus meetings at which experts present, discuss and agree recommendations.

- Initial drafting of guideline document which is circulated amongst experts and stakeholders for comments.
- Amendments incorporated and guidelines finalised.

- Guidelines piloted and evaluated.

- International dissemination.

The process has involved 49 experts from 8 countries reviewing between them, over 1000 papers. The WS guidelines are currently being piloted in 20 centres, by paediatricians and geneticists. The first draft of AS guidelines has been circulated for feedback. Consensus meetings for NS and KS will be held very shortly. The finished guidelines will be available from the DYSCERNE website.

\section{Author details}

'University of Manchester, The Nowgen Centre, University of Manchester, M13 9WU, UK. ${ }^{2}$ Center for Human Genetics, Herestraat 49, B-3000 Leuven, Belgium. ${ }^{3}$ Radboud University Nijmegen Medical Center, Department of Human Genetics 417, Geert Grooteplein 20, 6525GA, Nijmegen, The Netherlands. ${ }^{4}$ Istituto C.S.S. MENDEL, Unita' di Neurogenetica, Viale Regina Margherita 261, 00198 Roma, Italy. ${ }^{5}$ Département de Génétique Médicale, Hôpital de la Timone Enfant, 13385 Marseille cedex5, France. ${ }^{6}$ Department of Medical Genetics, The Children's Memorial Health Institute, Al. Dzieci Polskich 20, Warsaw 04-730, Poland.

Published: 19 October 2010

doi:10.1186/1750-1172-5-S1-P20

Cite this article as: Griffiths et al:: DYSCERNE: developing clinical

management guidelines for selected dysmorphic syndromes. Orphanet Journal of Rare Diseases 2010 5(Suppl 1):P20.

\footnotetext{
*Correspondence: pam.griffiths@cmft.nhs.uk

'University of Manchester, The Nowgen Centre, University of Manchester, M13 9WU, UK

Full list of author information is available at the end of the article
} 